\title{
El saber del maestro en contextos de diversidad socio-cultural: recuperación de las experiencias pedagógicas desde la interacción en red
}

THE WISDOM OF THE TEACHER IN THE CONTEXT OF SOCIO-CULTURAL DIVERSITY: RECOVERY OF PEDAGOGICAL EXPERIENCES FROM THE NETWORK INTERACTION

A SABEDORIA DO PROFESSOR NO CONTEXTO DA DIVERSIDADE SÓCIO-CULTURAL: A RECUPERAÇÃO DE EXPERIÊNCIAS PEDAGÓGICO PARTIR DA INTERAÇÃO IN REDE

Gloria Judith Castro Bohórquez*/gjcastro@unicauca.edu.co

Ulises Hernández Pino**/ulises@unicauca.edu.co

Resumen

El encuentro académico entre la universidad y la escuela para abordar los problemas de la educación, sigue siendo distante debido a las concepciones antagónicas de los maestros y los docentes universitarios. Este escrito socializa los aprendizajes en torno a la "Construcción de propuestas de innovación pedagógica en contextos de diversidad sociocultural desde la interacción en red" (VRI 2719), proyecto de investigación-acción en educación desarrollado a través del encuentro dialógico de maestros en formación, maestros en ejercicio y formadores de maestros.

\section{Summary}

The academic encounter between university and school to solve the problems of education is distant by antagonistic conceptions of teachers and university professors. This paper socializes the learning about the "Construction of educational innovation proposed in diverse sociocultural contexts through the network interaction" (VRI 2719), it's action-research project in education developed through dialogue between student teachers, practicing teachers and teacher trainers.

\section{Resumo}

O acadêmico desacordo entre a universidade ea escola para resolver os problemas da educação, é devido a concepções antagônico de professores e professores universitários. Este artigo apresenta aprendizagem sobre a "Construção da inovação pedagógica proposto em contextos da sócio-culturais diversidade a partir da interação in rede" (VRI 2719) projeto de pesquisa-ação em educação desenvolvidos através do encontro dialógico de professores em formação, professores em exercício e formadores de professores.

Grupo de Investigación en Enseñanza de las Ciencias y Contextos Culturales - GEC Red de Investigación Educativa ieRed Universidad del Cauca.

* Docente del Departamento de Educación y Pedagogía y Coordinadora del Grupo de Investigación en Enseñanza de las Ciencias y Contextos Culturales -GEC- de la Universidad del Cauca. Magíster en Educación con énfasis en Docencia de la Geografía de la Universidad Pedagógica Nacional.

** Asesor Académico del Contrato entre la Universidad del Cauca y Computadores para Educar para el desarrollo de la Etapa de Formación y Acompañamiento 2009 y 2010 en los Departamentos de Nariño y Putumayo. Candidato a Doctor en el Doctorado en Ciencias de la Educación del la Universidad del Cauca y Rudecolombia.

Fecha de recepción: 6 de agosto de 2011 / Fecha de aprobación: 8 de junio de 2012

26 nodos y nudos / volumen 4 N.. 32 / enero-junio 2012 / pp. 26-38 26 
Palabras clave

Formadores de maestros, saber pedagógico, experiencia pedagógica, diversidad socio-cultural, interacción en red

Keywords

Teacher trainers, pedagogical wisdom, pedagogical experience, socio-cultural diversity, network interaction.

Palavras-chave

Formadores de professores, sabedoria pedagógica, experiência pedagógica, diversidade sócio-cultural, a interação in rede

\section{El problema de investigación}

El trabajo de la Red de Investigación Educativa - ieRed 1 ha permitido demostrar que el encuentro académico entre la universidad y la escuela, para abordar los problemas de la educación preescolar, básica y media, sigue siendo distante debido a las concepciones que se ponen en juego entre los maestros y los docentes universitarios.

El maestro de educación preescolar, básica o media, tiene una amplia experiencia pedagógica desde lo cotidiano, por la necesidad de dar respuestas didácticas inmediatas a las múltiples situaciones que a diario surgen en el aula de clases. Sin embargo presenta debilidad en la argumentación y soporte conceptual, y en relacionar su actuar con las exigencias curriculares del Estado Colombiano. Mientras, por su parte, los docentes universitarios, en el ámbito de la formación inicial y en procesos de cualificación permanente de maestros, asumen esta orientación desde teorías universales y a través de un lenguaje formal, con lo que responden a lo requerido en las comunidades académicas pero perdiendo el vínculo con los contextos sociales específicos de las instituciones de educación básica y media.

A esto se suma que la preparación de maestros para todos los niveles se sigue pensando desde una idea de capacitación instrumental más que de formación, privilegiando la didáctica de corte eficientista y centrada en el aprendizaje (Zuluaga, et al., 1988, p. 5). Con esto se desconoce el valor de la subjetividad del maestro, que es donde de origina toda convicción y esfuerzo por trascender la labor educativa.

El compromiso de reconocer en el acto educativo a los otros como pares, es lo que posibilita construir relaciones humanas que favorecen la construcción de currículos acordes, tanto a una reflexión crítica sobre la realidad, como a la resignificación de las concepciones, los procesos y las prácticas pedagógicas en contextos y coyunturas concretas para su transformación (Unda, et al., 2001).

1 Iniciativa del Grupo de Investigación en Enseñanza de las Ciencias y Contextos Culturales -GEC- de la Universidad del Cauca, que busca la relación universidad-escuela, para abordar las problemáticas presentes en el aula de clases contemporánea y su relación con el contexto socio-cultural, a través del encuentro entre los maestros, docentes y profesores de los diferentes niveles educativos, con la pretensión de generar saber pedagógico de forma colectiva. 
Esta forma de entender la educación es especialmente importante en el Departamento del Cauca, en donde el $60 \%$ de la población es rural, hecho que contrasta el $75 \%$ de población urbana que tiene el resto del país. Además, el 19,6\% de la población es indígena y el $20,1 \%$ es afrodescendiente, situación que posiciona al Departamento como el segundo con mayor población indígena y el sexto en cuanto a población afrodescendiente en el país (PNUD, 2010, p. 14). Su línea de pobreza está por encima del $60 \%$, mientras que la línea de indigencia se encuentra por encima del 25\% (p. 25). Cifras que evidencian no sólo un alto nivel de diversidad étnica y cultural, sino también de condiciones que originan conflictos sociales.

Otra característica que es necesario destacar dentro de las configuraciones del Departamento, es que desde 1993 sólo ha tenido 1,6\% de crecimiento poblacional. Más allá de explicar este comportamiento demográfico en función de la disminución de la natalidad y el aumento de la emigración (PNUD, 2010, p. 16), se debe reflexionar acerca del impacto social y cultural que implica la disminución paulatina del número de habitantes en un territorio.

En este contexto, los maestros y las instituciones tienen el reto de abordar la educación básica y media, no sólo desde los planteamientos de las políticas educativas, sino también desde estas realidades. Pero estos retos no tienen solución si la actividad escolar se centra exclusivamente en los procesos evaluativos, que han llevado a convertir la mayoría de aulas de educación primaria en espacios de repetición, las aulas de educación secundaria en espacios de control coercitivo, y las aulas de educación media, en lugares poco proclives para potenciar las capacidades académicas y laborales de la población joven, desvirtuando el aula como espacio de relación entre maestros y estudiantes con el conocimiento y en los propios contextos.

Por lo tanto, la interacción en red22, en torno a la investigación en educación, de profesores universitarios (formadores de maestros), maestros de colegios (maestros en ejercicio) y estudiantes de normales o de licenciatura

\footnotetext{
2 La red, en este sentido, implica una forma de pensar y de actuar que busca el trabajo con otros para aunar esfuerzos con el fin de que cada uno alcance sus propósitos. Desde esta perspectiva el establecimiento de relaciones se hace a partir de una dimensión estratégica antes que colaborativa, ya que no necesariamente se comparte un mismo objetivo, y tampoco está relacionada exclusivamente con el uso de las TIC.
}

(maestros en formación), no sólo puede contribuir a la generación y recreación del currículo y de la pedagogía desde una perspectiva que implica una manera diferente de relación y de establecimiento de roles, sino que además se estaría avanzando en la consolidación de una comunidad académica alternativa, en donde la negociación de sentidos surge de la reflexión y la sistematización de los actores sobre su propia práctica y en diálogo con las teorías y las políticas educativas.

Se trata de cambiar la idea de trabajar sobre el currículo como la definición de actividades, contenidos y secuencias al margen de las personas y los contextos, por la de estar formándonos y apoyando la formación de otros mediante el currículo, de tal manera que las trayectorias personales, disciplinares e institucionales, señalen horizontes de sentido para el trabajo de construcción curricular, el desarrollo del conocimiento, la conformación de las profesiones y sus prácticas sociales (Gutiérrez y Perafán, 2002, p. 70).

Lo pedagógico, por su parte, no se reduce a la labor de saber aplicar en el salón de clase las teorías producidas por otras disciplinas científicas, perdiendo la posibilidad de pensar la relación maestro-escuela-sociedadEstado-cultura (Zuluaga, et al., 1988, p. 5), sino que en este nuevo espacio de reflexión y re-conceptualización que representa la red, se busca constituir la pedagogía como campo para pensar y actuar sobre la formación humana de lo humano, en el contexto de sus realidades sociales, económicas y culturales (Souza, 2007, p. 18).

Es así como se plantea el proyecto de investigación "Construcción de propuestas de innovación pedagógica en contextos de diversidad sociocultural desde la interacción en red" (VRI 27193), con el fin de promover reconceptualizaciones en las formas en que la escuela ha asumido la enseñanza de las áreas obligatorias y fundamentales de la educación básica y media, en el contexto particular del Departamento del Cauca.

\section{Referente metodológico del proyecto de} investigación

El referente metodológico escogido para el desarrollo de esta investigación fue el de Investigación- Acción en Educación, buscando con ello crear una dinámica de

\footnotetext{
3 Este proyecto fue seleccionado para su financiación en la VI Convocatoria de Apoyo a Proyectos de Investigación, Desarrollo Tecnológico e Innovación de la Universidad del Cauca del año 2009.
} 


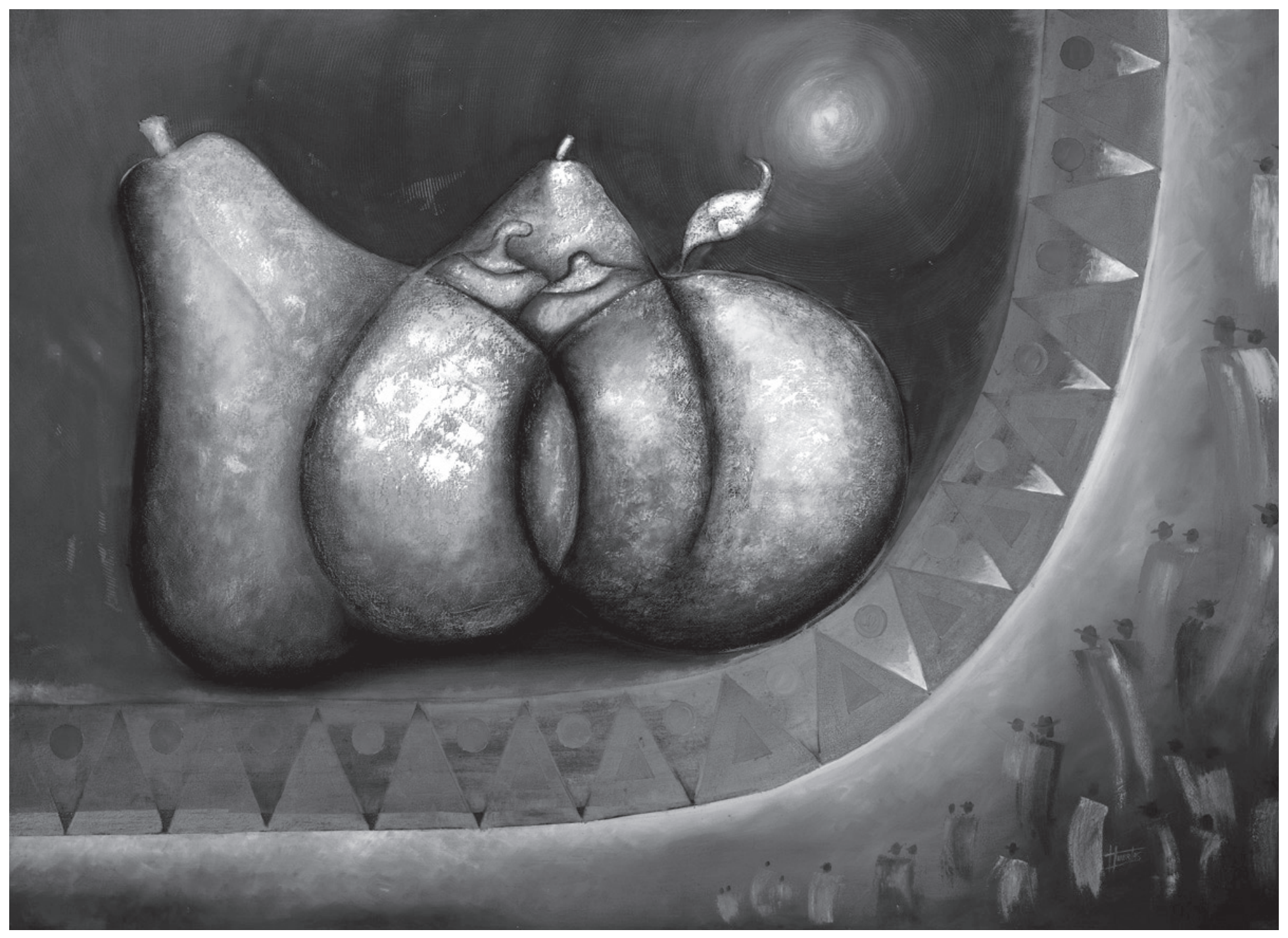

Segundo Arístides Huertas Torres ॥ Título: Bodegón onírico ॥Técnica: Óleo sobre tela ॥ Dimensiones: 140 cm x 160 cm 
participación y reflexión colectiva, en donde los maestros aborden sus problemáticas pedagógicas y generen propuestas educativas que les permitan transformar sus propias prácticas y dotar de sentido la enseñanza (Elliott, 2000, p. 25).

Con el fin de articular las posturas teóricas y metodológicas con las experiencias y vivencias de quienes participan en este proyecto, se constituyó un seminario permanente de reflexión, debate y síntesis, en el marco de la Red de Investigación Educativa -ieRed-, con la participación de maestros de Instituciones de Educación Básica y Media del Departamento del Cauca, profesores de la Escuela Normal Superior de Popayán, profesores y estudiantes de Licenciaturas en Educación Básica de la Universidad del Cauca e ingenieros que coordinan la cualificación de maestros en el suroccidente colombiano en el marco del programa Computadores para Educar del Ministerio de TIC.

Aunque en la planeación del proyecto se contemplaron cuatro momentos, el carácter flexible de la investigación-acción, que permite definir y redefinir las acciones en función de las decisiones que van tomando los participantes, hizo que el seminario realmente se desarrollara en tres grandes momentos, cada uno con dinámicas particulares y vinculadas a las características del seminario:

- Análisis: en donde los maestros en ejercicio socializaron las problemáticas centrales de su quehacer pedagógico y las acciones realizadas, lo que permitió la recuperación de la práctica del maestro como experiencia pedagógica.

- Debate: ya que el intercambio entre los maestros en ejercicio y los formadores de maestros en torno a las experiencias pedagógicas de los primeros, condujo a preguntarse por la pertinencia de los referentes teóricos del proyecto de investigación.

- Síntesis: al asumir el compromiso de dejar por escrito las socializaciones realizadas por los maestros en ejercicio, las reflexiones de los formadores de maestros y las experiencias iniciales de los maestros en formación.

Siendo consecuentes con el enfoque metodológico, a continuación se presentan los principales hallazgos y conclusiones que emergieron del trabajo realizado en el seminario.
Categorías de maestros para la consolidación del seminario como espacio de encuentro

Uno de los resultados del seminario fue la identificación de tres categorías para nombrar los diferentes grupos de maestros que participaban del proyecto, de tal modo que superara las clasificaciones excluyentes que se han presentado tradicionalmente entre los estudiantes y profesores de los programas de Licenciatura y las Escuelas Normales Superiores, así como entre estos y los profesionales no licenciados que trabajan en la educación básica y media, o los profesionales que orientan procesos de cualificación de maestros. Estas categorías fueron:

- Maestros en formación: para referirse a los estudiantes que se están formando, tanto en las Escuelas Normales Superiores, como en las Facultades de Educación.

- Maestros en ejercicio: para referirse tanto a los maestros licenciados, como no licenciados, que se preocupan por la formación integral de sus estudiantes.

- Formadores de maestros: para referirse tanto a los profesores encargados de la formación complementaria de las Escuelas Normales Superiores, como a los profesores universitarios que trabajan en los distintos programas de Licenciaturas, y a los profesionales de otras áreas que trabajan en programas o proyectos de formación de maestros.

Estas denominaciones también permitieron un autoreconocimiento de las fortalezas y debilidades de cada grupo de participantes, facilitando, de esta forma, el enriquecimiento de la interacción en el seminario desde las múltiples miradas que se potencian en estos espacios dialógicos, al situar a los distintos tipos de maestros en igualdad de condiciones para la interlocución.

El seminario permitió la expresión del maestro en formación, quien vive la dualidad de ser un estudiante que cuestiona la manera como está siendo formado, pero a quien a la vez se le dificulta tomar distancia de estas prácticas pedagógicas, las cuales tienen un peso muy fuerte en la definición de su ser como maestro. La importancia de la participación de los maestros en formación en el seminario radica en que ellos obligan 
a reflexionar sobre las contradicciones implícitas en el planteamiento del trabajo en el aula.

También participaron los maestros en ejercicio, quienes en el afán de atender las dinámicas absorbentes de la vida diaria de la escuela, como la llegada puntual, la vigilancia disciplinar, la restricción de la movilidad, el control de las posturas corporales y el manejo rígido de tiempos para las actividades, se ven imposibilitados para profundizar sobre los conceptos básicos de cada área escolar y su relación con la vida cotidiana. Estas situaciones no dan espacio para que piensen el sentido de la formación sobre la base de relaciones de respeto, reciprocidad y reconocimiento del otro. En este caso, el valor de su participación está en dar a conocer las situaciones que se presentan en el aula y la manera como se pone a prueba la inventiva y la creatividad para dar soluciones a las problemáticas que se presentan a diario en la escuela.

Los formadores de maestros, por su parte, viven la tensión de estar entre los conocimientos teóricos y los saberes prácticos. Al docente universitario se le exige el tratamiento de lo teórico como vía que hace posible la formación de licenciados, lo que en muchas ocasiones conlleva el distanciamiento de las realidades escolares. Para el profesor de las escuelas normales el predominio de la práctica conduce a la reproducción irreflexiva de prácticas pedagógicas. La existencia de estas tensiones muchas veces no es consciente, pero genera distanciamientos entre ambos enfoques, lo que no favorece el intercambio de experiencias, la interlocución fluida y la posibilidad de un trabajo conjunto.

\section{Fortalezas y dificultades de los maestros en la recuperación de sus Experiencias Pedagógicas}

El seminario de análisis, debate y síntesis del proyecto de investigación, inició con la solicitud a los participantes de la presentación de un escrito acerca de las dificultades que cada uno había tenido en la enseñanza de alguno de los cursos a su cargo. El texto elaborado se publicó en un espacio virtual para que la lectura y la realización de comentarios por parte de los demás participantes, se realizara antes de la socialización $y$, de esta manera, se enriqueciera con las preguntas, comentarios y observaciones.
La elaboración de los textos se fue realizando de forma paulatina y reflejó los diferentes tipos de dificultades. A los maestros les cuesta convertir sus historias y experiencias orales en textos escritos, asumir las observaciones como oportunidades para repensar su experiencia y valorar su experiencia cuando está escrita, por lo que sienten la necesidad de que sus documentos sean revisados por personas con autoridad teórica. El problema de este tipo de revisiones es que los textos pierden la voz del maestro al forzar los escritos en un formato legitimado académicamente, perdiendo la vitalidad de la experiencia en su relación con el contexto social. Esto sucede por la poca consciencia que hay entre los maestros en ejercicio de la potencia y el valor del saber pedagógico que poseen (Martínez, 2003, p. 186).

El seminario también permitió visibilizar la resistencia y el desconocimiento que muchos maestros tienen de las orientaciones curriculares que el Estado Colombiano ha formulado en las tres últimas décadas, para la enseñanza de las distintas áreas en la educación preescolar, básica y media. Lo que les impide clarificar cómo la experiencia pedagógica responde a las políticas educativas, o cómo se distancia y plantea algo diferente.

Uno de los elementos a rescatar del seminario, fue la generación de vínculos académicos entre maestros en ejercicio y formadores de maestros en torno a las experiencias pedagógicas, porque fueron disminuyendo los niveles de prevención a la crítica, y porque el seminario era prueba de la posibilidad de hacer realidad los nexos entre instituciones de educación básica y media, escuelas normales y programas de licenciaturas en calidad de pares.

La necesidad de preparar un escrito para participar del seminario ocasionó que el número de maestros participantes se fuera reduciendo con el tiempo; los maestros que permanecieron, fueron aquellos que ya venían adelantando acciones para abordar problemáticas previamente identificadas, encontrando en el seminario el espacio ideal para socializar, discutir y sistematizar lo que venían reflexionando y haciendo. Por ello fue importante que el criterio de valoración no fuera sólo el nivel logrado en los escritos, sino la disciplina y el ejercicio constante de ordenación y reordenación con base en los comentarios planteados por los demás participantes. 


\section{Aulas que reflejan la diversidad social y cultural}

En este primer momento del seminario se logró la identificación de las problemáticas pedagógicas en diferentes áreas de educación básica, las cuales fueron la base para la realización del I Seminario-Taller de Experiencias Pedagógicas en el Aula, realizado en la Escuela Normal Superior de Popayán, en septiembre del 2010. Al hacer un análisis de las presentaciones se encontró que el concepto de aula no estaba circunscrito al salón de clase (Cerda, 2002, p. 15), y que este tipo de investigaciones se centra en el ámbito micro de la educación (Buenaventura, 2000, p. 29), lo cual se complejiza cuando se aborda desde el reconocimiento de la diversidad de sus múltiples facetas: étnica, de género, lingüística, social y cultural.

En la presentación "La narración itinerante: nárranos tu cuento, cuéntanos tu historia", se recupera la Tulpa como el espacio formativo ancestral de las comunidades indígenas del Cauca, porque alrededor del fogón, y desde lo oral, se transmite la cultura de los mayores a los jóvenes, se organiza el pensamiento para asegurar la existencia como pueblos y se aconseja desde el modo de ser Nasa (Comunidad de los Paeces) o Misak (Comunidad de los Guambianos). En este contexto el aula escolar es el espacio de encuentro entre culturas, espacio del que las comunidades indígenas han apropiado para hacer realidad una pedagogía intercultural bilingüe, desde el diálogo de saberes y la confrontación de cosmovisiones diferentes.

El Aula Comunitaria es aquella que se forma por fuera de las estructuras físicas de las Instituciones Educativas. Es así como "la enseñanza del álgebra mediante la preparación de una taza de café" es una presentación que vincula una clase de matemáticas con prácticas culturales que se realizan desde los núcleos familiares de los indígenas de la comunidad Nasa de Novirao, como una clara expresión de la construcción de abstracciones a partir de lo concreto.

A su vez "La enseñanza de la Química a través del estudio de plantas tradicionales" como experiencia presentada por una institución educativa urbana y de carácter privado, mostró la forma de promover formas organizativas arraigadas en sectores indígenas, afrodescendientes y campesinos, desde el área de Ciencias Naturales, despertando el interés de los estudiantes por la indagación del entorno social, cultural y ambiental de su región.

El Aula Multimedial, por su parte, irrumpe con fuerza para conectar lo local con lo global, en un intento por aprovechar la apropiación que los jóvenes hacen del ciberespacio, con todas las ventajas y desventajas que este ejercicio propicia. Claro ejemplo de ello fue la presentación "De la grabadora de mi mamá, al documental en digital: los aprendizajes en Ciencias Sociales mediados por las TICs" que hace uso de estas tecnologías para la recuperación de testimonios familiares sobre los sucesos ocurridos el 9 de abril de 1948.

\section{Debate de los referentes teóricos del proyecto de investigación}

Un segundo momento del seminario consistió en abordar el debate sobre aspectos que fueron surgiendo en las socializaciones y discusiones de los maestros, y que cuestionaban los fundamentos del proyecto de investigación. Para abordar estos debates se programaron sesiones del seminario en donde se contaba con dos ponencias preparadas por los mismos participantes, pero desde posturas diferentes. El primer elemento debatido en profundidad, giró en torno a la innovación pedagógica. Al respecto, Francisco Aguilar (1998) plantea que: "una determinada realidad educativa puede ser caracterizada como innovadora, sólo en referencia a otra realidad educativa conocida que se considera como tradicional o convencional y frente a la cual la nueva propuesta se ofrece como una alternativa diferente" (p. 127).

Sin embargo, la dinámica del seminario no permitía asegurar que los maestros en ejercicio estuvieran hablando de innovaciones pedagógicas. En primer lugar, porque los relatos hacían referencia al análisis de situaciones de aula teniendo como eje central el contexto, y no necesariamente los problemas didácticos de la enseñanza de un área en particular. En segundo lugar, porque no había el interés de comparar las experiencias entre sí para mostrar su grado de novedad y singularidad, ya que su valor estaba en la forma como habían interrelacionado, de forma consciente o no, el contexto socio-cultural, el desarrollo temático del área y las situaciones problemáticas que se presentaban en el aula. Así que, con el surgimiento de los primeros borradores de escritos, se encuentra que lo que allí se empieza a plasmar son unas experiencias pedagógicas. 
La dinámica de socialización, discusión y escritura, de la propia experiencia en el seminario, lograba que el maestro iniciara un proceso sistemático de reflexión y producción de saber, para lo cual era necesario considerar otros referentes como los de la Expedición Pedagógica Nacional, que plantean que:

Ese paso de portador a productor de saber, se da sólo a condición de que el maestro al reflexionar su práctica, la convierte en experiencia. La experiencia es, según Dewey la recuperación de un saber que nos antecede. Ello significa, que una práctica se convierte en experiencia, sólo cuando aquella es pensada, esto es, cuando se reconoce como producto de un saber o de una cultura (Martínez, et al., 2002, p. 92).

El segundo aspecto del debate que se discutió en profundidad, giró alrededor del referente metodológico. Si bien el proyecto de investigación se planteó desde la Investigación-Acción en Educación, las experiencias pedagógicas mostraban un trabajo de indagación y de sistematización más próximo a la Investigación en el Aula. La razón por la que se planteó en el proyecto la Investigación-Acción en Educación, fue que se buscaba impulsar que fuera el propio maestro quien profundice en la comprensión de los problemas de aula, desde su experiencia pedagógica y en relación con la literatura académica, y fuese capaz de describir, delimitar y actuar sobre dichos problemas con el análisis y reflexión que pueda hacer con otros maestros.

Por su parte, la Investigación en el Aula es una perspectiva que introduce en Colombia, durante la década de los ochenta, la idea de maestro investigador, particularmente con el trabajo de Eloísa Vasco (1988), y que busca que el maestro aborde las problemáticas del aula de forma sistemática a través de la delimitación de un problema, la descripción de la forma en que se podría resolver y la formulación de una propuesta pedagógica, para darle una solución (p. 100).

En este caso se concluyó que ambos enfoques de investigación estaban presentes en el desarrollo del proyecto de investigación, aunque en niveles diferentes. Mientras la Investigación en el Aula permite orientar el trabajo de indagación y sistematización del maestro en el aula para la producción de saber pedagógico, la Investigación-Acción en Educación, permite la articulación y el crecimiento colectivo de las experiencias de cada maestro participante en el seminario.
En este segundo momento del seminario se abordaron otras discusiones, aunque sin la misma profundidad que las dos primeras, pero que plantearon la necesidad de considerar una segunda fase del proyecto de investigación para abordar estos aspectos con mayor rigurosidad. Una de estas discusiones se dio alrededor de las expresiones "educación en la diversidad" e "inclusión educativa". Las diversas posturas y experiencias de los participantes hicieron evidente la necesidad de develar la intencionalidad y las consecuencias de estos discursos en las instituciones de educación básica y media.

Otra de las discusiones se dio en torno a los "contextos de diversidad socio-cultural". Las experiencias pedagógicas mostraron que las realidades del Departamento del Cauca tienen diferentes contextos sociales, pero que al interior de cada uno de estos, coexisten variadas prácticas culturales atravesadas por lo étnico, lo lingüístico, lo religioso, entre otras situaciones. Esta condición llevó a identificar que uno de los retos es indagar y profundizar en el significado de las expresiones "contexto social" y "prácticas o expresiones culturales".

La discusión también tocó el tema de enseñanza de las ciencias, y dentro de este una de las observaciones que tuvo mayor relevancia fue la del reconocimiento de distintas formas de aproximarse a la realidad desde el conocimiento, donde interactúan de manera simultánea varias visiones de mundo que resultan ser antagónicas en su desarrollo. A modo de ejemplo, cabría preguntarse cómo enseñar el ciclo hidrológico en comunidades campesinas e indígenas cuyas concepciones cosmogónicas le dan al trueno, al rayo y al relámpago una designación de carácter espiritual. En este caso entran en confrontación las ideas de la existencia misma de un pueblo en la interpretación de los fenómenos naturales y las precisiones de carácter físico y químico.

Por tanto, el reto de la educación preescolar, básica y media, no consiste en transponer conceptos derivados de la ciencia en el desarrollo de las clases, sino en la integración de las áreas, para ganar en reconocimiento y comprensión de las diferentes situaciones sociales y ambientales que están presentes alrededor de la escuela. Aunque se pusieron en evidencia diversos enfoques para aproximarse al debate, no hubo un direccionamiento único en donde se buscara hacer prevalecer una postura sobre las demás, sino que el ejercicio tuvo como bondad la ampliación de la mirada y la construcción de posturas, unas conjuntas y otras individuales, desde la discusión dialógica. 


\section{La escritura como camino para el surgimiento del saber pedagógico}

El tercer momento del seminario se enfocó en la producción escrita, con el propósito de generar memoria de las experiencias pedagógicas de los diferentes participantes, así como de las discusiones realizadas en el seminario. Sin embargo, se entendió que cada grupo de maestros tenía realidades, niveles y capacidades diferentes desde donde se podrían plantear los escritos, por lo que se planteó la proyección de la publicación de un libro con estas experiencias, para considerar la producción escrita de los maestros en formación, los maestros en ejercicio y los formadores de maestros.

\section{Experiencias iniciales de los Maestros en} Formación en su Práctica Pedagógica

La posibilidad de vincular al proyecto de investigación a estudiantes del programa de Licenciatura en Educación Básica, convocados bajo la figura de semillero de investigación, inició un trabajo de recuperación de sus experiencias como estudiantes y maestros, debido a que se dio la coincidencia de que el grupo interesado estaba compuesto por egresados de la formación básica complementaria de algunas Escuelas Normales del Departamento del Cauca.

Los textos que surgieron de esta experiencia, dan cuenta de la aproximación que los maestros en formación han hecho de las realidades del aula de clase, a través del desarrollo de las Prácticas Pedagógicas Investigativas. Es así como el escrito "La Normal de Belalcazar: huella imborrable en los procesos formativos de maestros", deja entrever el impacto causado por la avalancha del Río Paéz en los procesos formativos de maestros, en un contexto social que aglutina prácticas culturales indígenas, afrodescendientes y campesinas en la Escuela Normal de Tierradentro.

Los escritos "La carta informal: estrategia para perder el miedo a escribir" y "Las canciones subtituladas facilitan la adquisición de vocabulario en inglés", presentan formas para potenciar el desarrollo de las habilidades comunicativas a través de estrategias tendientes a superar los miedos en el momento de escribir. Por otra parte, "El ábaco vertical: una manera creativa de aprender matemáticas" es un escrito que recrea la historia del crecimiento urbano de la ciudad de Popayán, y pone resalta la importancia de las relaciones de convivencia de la comunidad escolar y su alto sentido de pertenencia al lugar.

Finalmente, con "Los pactos de aula: otra manera de enseñar y aprender ciencias naturales", se tiene un escrito que narra las vicisitudes de un maestro en formación que se pregunta por las interacciones sociales, tanto con la maestra asesora, como con los niños, y por las relaciones con el conocimiento escolar desde el reconocimiento de sus propias debilidades. Ante la necesidad de generar otras dinámicas escolares, encuentra en las salidas de observación una manera de irrumpir en el mundo de la educación ambiental.

En estos escritos los maestros en formación ponen en evidencia los miedos al momento de enseñar, las dificultades con los niños, las deficiencias en torno al manejo de conceptos básicos, al igual que las fortalezas y los aciertos que surgen de ellos mismos, lo cual les permite generar propuestas creativas que se hacen evidentes a través de los escritos.

\section{Experiencias en contextos de diversidad de los} Maestros en Ejercicio

Por otro lado están los escritos de los Maestros en Ejercicio, los cuales buscaron recoger la experiencia tendiente a solucionar problemáticas específicos se su práctica pedagógica. Por ejemplo, en el escrito "La enseñanza del álgebra mediante la preparación de una taza de café", un maestro encuentra la manera de trabajar el tema de las ecuaciones algebraicas de primer grado en un curso de octavo, con las vivencias de los estudiantes en una comunidad indígena, a partir de la preparación de una taza de café.

De la misma forma, "La narración itinerante: nárranos tu cuento, cuéntanos tu historia", crea una cátedra narrativa itinerante, como la manera de establecer las relaciones entre el desarrollo de las habilidades comunicativas en el área de lengua castellana, y la recuperación de la tradición oral de la Comunidad Nasa. Es importante resaltar la proyección que tuvo este trabajo pedagógico en un contexto de diversidad lingüística, como aporte de los estudiantes de grado noveno a los procesos de enseñanza de la lengua castellana en las sedes de educación básica primaria en corregimientos vecinos.

"La huerta como laboratorio vivo: propuesta pedagógica para la enseñanza de las ciencias", es un escrito que aborda la enseñanza de la química en una institu- 


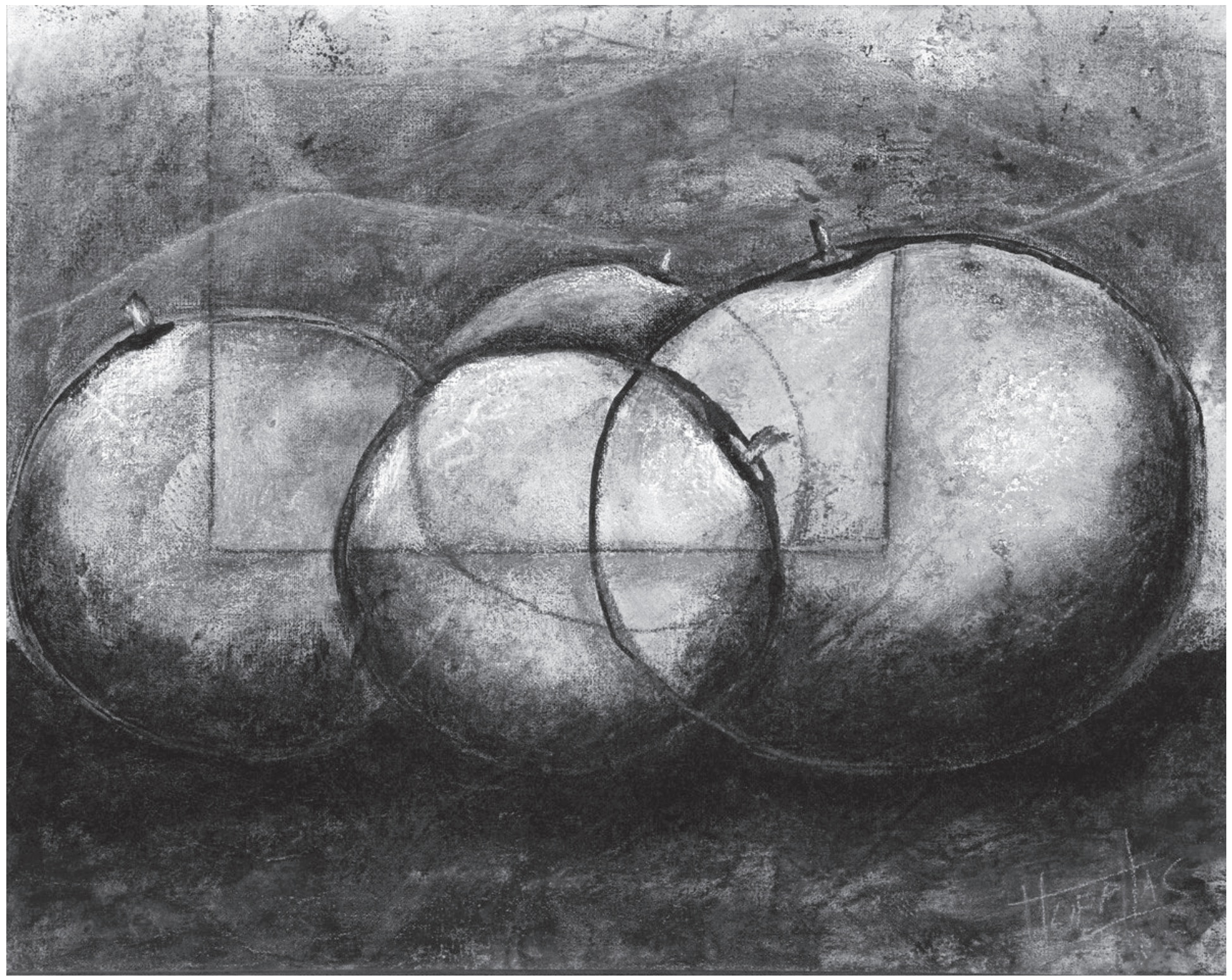

Segundo Arístides Huertas Torres ॥ Título: Bodegón »Técnica: Mixta sobre tela ॥ Dimensiones: $50 \mathrm{~cm}$ x $70 \mathrm{~cm}$ 
ción de carácter privado, y recupera una experiencia investigativa sobre plantas medicinales en el Resguardo de Tierradentro, pero además se convierte en un espacio para reflexionar sobre las dificultades que tiene la preparación separada de los maestros de ciencias naturales, en las áreas de biología, química y física.

Por último, vale la pena señalar el texto de "Compartir experiencias entre maestros: una forma de hacer didáctica en inglés", que narra la experiencia de una Licenciada en Educación Básica con Énfasis en Lengua Castellana e Inglés, que se incorpora a un programa universitario de extensión para la enseñanza del Inglés como lengua extranjera, para la formación en didáctica, de los instructores de este programa.

\section{La producción de saber pedagógico de los Formadores de Maestros}

Los escritos de los Formadores de Maestros se plantearon con el fin de conocer las propuestas pedagógicas y curriculares de formación, y las reflexiones de orden teórico y práctico, que han generado los participantes del seminario en el ámbito de las Escuelas Normales Superiores, de las Licenciaturas en Educación Básica y de los programas gubernamentales para la cualificación de maestros.

En cuanto a la formación de normalistas superiores, la experiencia "Aproximación a una configuración didáctica para la lectura y escritura del contexto", relata el proceso de reconfiguración didáctica de los maestros en formación para la enseñanza de la lectura y escritura de niños de primer grado, partiendo de las habilidades y destrezas de los normalistas, lo que pone en evidencia la creatividad y el compromiso para abordar un proceso escolar tan importante como la adquisición de la lengua escrita.

La otra experiencia, "La enseñanza y el aprendizaje de las ciencias desde los proyectos de aula", hace un análisis de la noción de ciencia en las orientaciones curriculares que el Estado Colombiano hace a los maestros de los niveles de pre-escolar y educación básica y media. Además, el escrito aboga por la integración de las diferentes áreas escolares, teniendo como eje la Práctica Pedagógica Investigativa orientada por un colectivo de profesores, desde la consciencia de la responsabilidad que tienen en relación con las sedes educativas con las cuales trabajan.
Cabe resaltar la importancia del seguimiento minucioso que se realiza en la experiencia "Las TIC como estrategia didáctica alternativa para afianzar procesos de formación autodirigida en la enseñanza de inglés como lengua extranjera", que presenta el reto de la adquisición del inglés como lengua extranjera en un contexto altamente monolingüe, y la formulación didáctica que de ella se deriva al hacer la inserción de las TIC con niños, adolescentes y jóvenes, inmersos en la cultura digital, pero con enormes resistencias para asumirla en los espacios académicos.

En el ámbito de la formación de maestros en los programas de Licenciatura en Educación Básica, se presenta la experiencia "Sociedad, cultura y educación: una experiencia relacional en la formación de licenciados en educación básica", que da cuenta detallada de las problemáticas que tienen los estudiantes, al ingresar a la universidad, en relación con textos especializados en el campo de la enseñanza de las ciencias humanas y sociales. A la vez, presenta de manera descriptiva las maneras como los estudiantes interpretan dichos textos y las posturas que asumen en relación con los autores.

Finalmente, se presenta la experiencia "Para qué las TIC en la Educación Básica y Media: reflexiones a partir de la cualificación de maestros en ejercicio en el suroccidente colombiano", cuyo aporte consiste en mostrar las distancias entre los discursos y políticas oficiales de inserción de estas tecnologías en el aula de clases, y las realidades con las que los formadores de maestros se encuentran al trabajar en los sitios más recónditos del país. A su vez, presenta las limitaciones y posibilidades de estos espacios de formación para repensar las prácticas pedagógicas en la tensión entre lo global y lo local, y una mirada que recupera las iniciativas de los maestros en medio de situaciones de adversidad.

Estas reflexiones fueron la base para la realización del II Seminario de Experiencias Pedagógicas de Aula, realizado durante el mes de junio de 2011, en la Escuela Normal Superior "Los Andes" en la Vega (Cauca). En este espacio se abrió la posibilidad de realizar eventos itinerantes por el Departamento, con el propósito de aunar lazos académicos entre la universidad y las escuelas normales para pensar, discutir y actuar, sobre los problemas educativos en contextos de diversidad socio-cultural. 


\section{Algunas conclusiones}

El seminario de análisis, debate y síntesis abrió la posibilidad de:

- Materializar las posibilidades de la interacción en red en los procesos de formación de maestros, en la medida que posibilitó plasmar el pensamiento en un escrito que daba cuenta de las posturas teóricas y su articulación con las aulas de clase en su cotidianidad.

- Reconstruir la experiencia pedagógica en cuanto a sus actividades, aprendizajes y dificultades. La necesidad de presentar con rigor lo planteado, implicó un ordenamiento de las ideas en torno a la experiencia pedagógica y el reconocimiento de las ideas o posturas teóricas que la fundamentan.

- La revisión de los escritos por parte de los colegas, generó un diálogo formativo a dos niveles: el encuentro de pares orientado a la corrección del documento y la socialización de las observaciones frente al grupo, lo cual no sólo significó aportes para quien escribía, sino para el resto de los participantes, al generar inquietudes y preguntas sobre los propios escritos en un ambiente de respeto, confianza y mutuo reconocimiento.

- La interlocución constituyó un factor de cohesión social que generó sentidos de pertenencia, cualificación pedagógica y una ganancia en madurez, en cuanto a la aceptación de comentarios críticos respecto de la producción.

- El fortalecimiento de los vínculos de colegaje a partir del proceso de revisión conjunto de los textos, permitió la interacción fluida entre los participantes, independientemente del nivel educativo en el cual se desenvuelven o de su titulación.

Así mismo, no se puede desconocer la necesidad de mejorar en algunos aspectos en los que notamos dificultades. Entre ellos:

- La urgencia de ganar dominio en el manejo de las Tecnologías de la Información y las Comunicaciones (TIC) para establecer otros momentos, canales y espacios de comunicación efectiva con los colegas, debido a los cruces de horarios, compromisos institucionales o distancias geográficas, aspectos que dificultan tener espacios de encuentro en un mismo horario y lugar.

- La necesidad de valorar el propio trabajo para vencer los miedos e inseguridades que tenemos los maestros al escribir, y que se ven reflejados en la tendencia a asimilar las observaciones y críticas como invalidación de las ideas y no como aspectos puntuales a revisar.

- La lectura minuciosa de los documentos sobre orientaciones curriculares que el Estado Colombiano ha generado en las últimas tres décadas, ya que en ellos se encuentran temáticas, estrategias didácticas, procesos de aprendizaje, enfoques pedagógicos y criterios evaluativos que le permitirían al maestro ratificar, innovar, e incluso, transformar sus prácticas pedagógicas a la luz de unos referentes académicos serios y sistemáticos.

\section{Agradecimientos}

El desarrollo de este proyecto de investigación fue posible gracias a la decidida participación de los maestros en formación Julieth Magally Atillo, Yenny Paola Gutiérrez, Luis Orlando Serna, Luz Janet Dorado, María del Carmen Cifuentes, Erika Palechor Jiménez, Ruber Higón Jiménez y Abel Angulo Agredo, estudiantes del programa de Licenciatura en Educación Básica de la Universidad del Cauca. Además, es necesario hacer un reconocimiento a los maestros en ejercicio Clara Stella Alzate de la Institución Educativa Marden Arnulfo Betancourth, Martha Teresa López del Real Colegio San Francisco de Asís, Edwin Andrés Murillo de la Institución Educativa Novirao, Sandra Lorena Moreno del programa Unilingua de la Universidad del Cauca y Oriana Robles Muñoz de la empresa Racore Ingeniería Divertida. Sin desconocer el aporte de los formadores de maestros Olga Marlene Campo, Henry Vargas y Luis Alberto Cuéllar, de la Escuela Normal Superior de Popayán; María Andrea Simmonds, Yoner Fernando Campo y Pastor Benavides, de la Universidad del Cauca; y a William Fernando Fernández del SENA Seccional Cauca. Así como al apoyo recibido en la VI Convocatoria a Proyectos de Investigación, Desarrollo Tecnológico e Innovación de la Universidad del Cauca. 
Aguilar, F. (1998). Innovaciones educativas y culturas contemporáneas. La investigación: fundamento de la comunidad académica. Bogotá: Instituto para la Investigación Educativa y el Desarrollo Pedagógico.

Buenaventura, N. (2000). La investigación como práctica pedagógica. Trabajo presentado en el Simposio Internacional de Investigadores en Educación. Santa Marta: Convenio Andrés Bello y Centro de Estudios e Investigaciones Pedagógicas.

Cerda, H. (2002). El proyecto de aula: el aula como un sistema de investigación y construcción de conocimientos. Bogotá: Magisterio.

Elliott, J. (2000). La investigación-acción en educación. Madrid: Morata.

Gutiérrez, E. F., y Perafán, L. (2002). Currículo y práctica pedagógica. Popayán: Universidad del Cauca.

Martínez, A. (2003). La enseñanza como posibilidad de pensamiento. En Zuluaga, O., et al. (Eds.), Pedagogía y Epistemología. Bogotá: Magisterio.

Martínez, A., Unda, M. P., y Mejía, M. R. (2002). El itinerario del maestros: de portador a productor de saber pedagógico. En
Suárez, H. (Comp.). Veinte años del movimiento pedagógico 1982-2002: entre mitos y realidades. Bogotá: Magisterio, 61-94.

Programa de las Naciones Unidas para el Desarrollo, PNUD. (2010). Los Objetivos de Desarrollo del Milenio y los desafíos del cambio climático en el departamento del Cauca. Bogotá. Obtenido en junio de 2011, desde, http://www.odm.pnudcolombia. org/raiz_odm2/cauca_odm.pdf

Souza, J. (2007). La contribución del pensamiento de Orlando Fals Borda a la teoría de la educación. En Del Campo, B. (Ed.). Investigación acción y educación en contextos de pobreza. Bogotá: Universidad de La Salle, 15-35.

Unda, M. P., Orozco, J. C., y Rodríguez, A. (2001). Una construcción colectiva de conocimiento pedagógico en la Expedición Pedagógica Nacional: huellas y registros. Bogotá: Universidad Pedagógica Nacional.

Vasco, E. (1988). La investigación en el aula: una alternativa. Revista Investigación Educativa, 5.

Zuluaga, O., Echeverry, A., Martínez, A., Restrepo, S., y Quiceno, H. (1988). Educación y pedagogía: una diferencia necesaria. Revista Educación y Cultura, 14.

\section{Diálogo del conocimiento}

El presente artículo presenta un análisis de las transformaciones que se promueven en la incorporación de la red al sistema educativo haciendo énfasis en alguna dimensiones tales como: las peculiaridades de la práctica pedagógica, la vinculación de la comunidad y el territorio e, implícitamente, las maneras de organización y gestión de las instituciones educativas en contextos de diversidad socio-cultural. En este sentido, la mirada social que se hace de la red forma parte de lo que se puede considerar el compromiso de la recuperación de las experiencias pedagógicas en la transformación de la sociedad a través de construir propuestas de innovación pedagógica en las que se integran aspectos como la alfabetización digital, la formación de maestros en ejercicio y en formación para el uso y manejo de la información, el cultivo de la lectura y la escritura como posibilidad de reflexión dela práctica pedagógica, el desarrollo de la comunicación y el examen de las dimensiones del aprendizaje y las tensiones existentes entre los conocimientos teóricos y los saberes prácticos que permiten: de un lado, la producción de saber pedagógico, y de otro lado, el aprendizaje a lo largo de la vida.

Las características señaladas pone en evidencia el desarrollo de métodos, didácticas y herramientas de análisis de entornos de interacción de grupos diversos que favorecen formas de colaboración para el aprendizaje basadas en intereses comunes y no en la coincidencia geográfica sino en la riqueza de la diversidad social y cultural del Departamento del Cauca. Por ello, la experiencia contempla la forma de mejorar el uso de la red en lo que se puede denominar la configuración de buenas prácticas de trabajo colaborativo académico en entornos virtuales, en donde se dinamiza el currículo, se cualifica el profesor, el estudiante, se identifican necesidades de aprendizaje y se diseñan alternativas de evaluación de los espacios de interacción para, finalmente, retroalimentar el análisis de las interacciones de los usuarios de la red.

Roberto Medina Bejarano 